\title{
Compression in wearable sensor nodes: impacts of node topology
}

\author{
Syed Anas Imtiaz, Student Member, IEEE, Alexander J. Casson, Member, IEEE, and \\ Esther Rodriguez-Villegas, Senior Member, IEEE
}

\begin{abstract}
Wearable sensor nodes monitoring the human body must operate autonomously for very long periods of time. Online and low power data compression embedded within the sensor node is therefore essential to minimise data storage/transmission overheads. This paper presents a low power MSP430 compressive sensing implementation for providing such compression, focusing particularly on the impact of the sensor node architecture on the compression performance. Compression power performance is compared for four different sensor nodes incorporating different strategies for wireless transmission/on-sensor-node local storage of data. The results demonstrate that the compressive sensing used must be designed differently depending on the underlying node topology, and that the compression strategy should not be guided only by signal processing considerations. We also provide a practical overview of state-of-the-art sensor node topologies. Wireless transmission of data is often preferred as it offers increased flexibility during use, but in general at the cost of increased power consumption. We demonstrate that wireless sensor nodes can highly benefit from the use of compressive sensing and now can achieve power consumptions comparable to, or better than, the use of local memory.
\end{abstract}

Index Terms-Compressive sensing, MSP430, low power consumption, wearable medical sensors, body area networks, EEG.

\section{INTRODUCTION}

$\mathbf{H}$ IGHLY miniaturised wearable sensors promise to allow the unobtrusive, convenient, and long term monitoring of a range of physiological parameters [1]. Their objective is to provide high quality, long term out-patient recordings of body functions such as the EEG (electroencephalogram), EOG (electrooculogram), EMG (electromyogram), and ECG (electrocardiogram), to facilitate improved disease diagnosis, closed loop treatment devices, and a shift towards personalised and preventative healthcare [2]. For end users the key parameters are: accuracy, minimal discomfort, and ease of use.

The power consumption of the sensor node is a critical factor in realising all of these. The current draw of the sensor sets the physical size of the battery required, which determines the device size and operating lifetime, which in turn affects the

Manuscript received $31^{\text {st }}$ January 2013. Revised $9^{\text {th }}$ September 2013 and $22^{\text {nd }}$ November 2013. Accepted $26^{\text {th }}$ November 2013.

S. A. Imtiaz and E. Rodriguez-Villegas are with the Circuits and Systems Group, Electrical and Electronic Engineering Department, Imperial College London, UK email: (\{anas.imtiaz,acasson,e.rodriguez\}@imperial.ac.uk).

A. J. Casson is with the Imaging and Signal Processing Group, School of Electrical and Electronic Engineering, The University of Manchester, UK email: (alex.casson@manchester.ac.uk)).

The research leading to these results has received funding from the European Research Council under the European Community's $7^{\text {th }}$ Framework Programme (FP7/2007-2013) / ERC grant agreement no. 239749. ease of use. It is widely accepted that the power consumption of sensor nodes can potentially be reduced by the inclusion of online, real-time, data compression embedded in the node itself [2]-[4]. The challenge lies in having compression algorithms that provide a high level of data reduction while introducing little error into the recorded signal and which require very little power to operate.

This paper presents a Texas Instruments MSP430 based sensor architecture for providing such compression, and in particular investigates the impact of the overall sensor node topology on the compression performance. Both wireless sensor nodes, where the sensor incorporates a wireless transmitter for passing the collected data to a processing smart-phone or computer in real-time; and local memory sensor nodes, where memory incorporated in the sensor is used to store the data until the node is physically connected to the processing computer; are widely used sensor node topologies. However these two different types of back-end have widely different requirements, constraints and impacts on the compression to be used. We investigate performance in terms of both reconstruction accuracy and power consumption in four different sensor nodes, two from each topology type, and provide a real system comparison of state-of-the-art compression for sensor nodes.

Compressive sensing is used as the compression basis as it is a relatively new technique very suitable for use in power constrained sensor nodes: the computational complexity of the different compression stages naturally fit within the power budgets available in the different stages of wearable sensor systems [5]. The fundamental compression step is simply a random sampling of the input signal and so has a low complexity for running online in the very power constrained sensor node. This low computational complexity is traded-off with having a higher complexity when the input signal needs to be reconstructed from the compressed samples. However this stage can be run on a smart-phone or fixed computer installation with much relaxed power constraints compared to the sensor itself. Compressive sensing itself can be implemented in either analogue or digital domains. In the former case, an ADC with a sub-Nyquist frequency is used to reduce sampling rate (and consequently reduce data) while the latter uses a standard Nyquist ADC followed by a sparse sensing matrix for data reduction. A complete comparison of these two compressive sensing implementations and their merits is discussed in [6]. In this paper, compressive sensing in digital domain is used for EEG signals that are generally sampled at low frequencies in the range 200-1000 Hz. Since these 
sampling rates are easily achievable, no further reduction in sampling frequency is desired (which is the main motivation of using analogue-domain compressive sensing) hence digital compressive sensing is preferred for data reduction.

The underlying theory of compressive sensing has been established previously [7], [8], as has the reconstruction performance in a number of applications [9]-[14]. We now evaluate compressive sensing in terms of both reconstruction performance and power consumption of the system including the back-end. We thus demonstrate, using two completely different wireless transceiver configurations, that compressive sensing is highly beneficial for wireless sensor nodes. These now achieve power consumptions comparable to, or better than, local memory based systems. Further, we find the reconstruction performance is largely independent of both fixed/floating point realisations and processing frame size choices. As a result these choices are dominated by, and must be correctly be chosen, based upon the hardware back-end used; not upon only signal processing considerations as previously thought. Previous studies have investigated compressive sensing performance in terms of reconstruction accuracy only, or only considering one back-end technology, and as a result have not been able to establish this critical factor for the practical application of the technique over a range of sensor nodes.

Section II outlines our four prototype sensor nodes, all of which are MSP430 based, with this being connected to a wireless transmitter on the same chip, an external wireless transmitter chip, a NAND flash memory chip, and a microSD card incorporating a FAT16 file system. Section III details the performance assessment of these different nodes where we use scalp EEG signals as an example medical application. Section IV presents detailed results for the performance of different compressive sensing configurations in terms of both reconstruction accuracy and power consumption, and these are discussed in Section $\mathrm{V}$ to provide an overview of the relative performance, advantages, and disadvantages, of the different sensor node topologies.

\section{SENSOR PLATFORMS}

\section{A. Hardware set up}

Due to its ultra low power consumption the Texas Instruments MSP430 family [15] is a popular hardware platform for wearable sensor nodes (for example it is used in the TinyNode, Tmote Sky, and Shimmer systems [16]-[18]) and it is the basis of our sensor nodes. Three of our prototypes use the MSP430F5438A micro-controller connected to different back-end systems as discussed below, while the fourth uses the CC430F6147. This is a single system-on-chip solution combining both an MSP430 core and a wireless transceiver on the same microchip allowing significant miniaturisation and cost savings. The set up of these four systems is shown in Fig. 1.

We have implemented four different prototypes across the two wireless and local memory topologies using:

1) A Nordic nRF2401+ wireless transmitter.

2) A Texas Instruments $\mathrm{CC} 430$ wireless transmitter.

3) A microSD card.

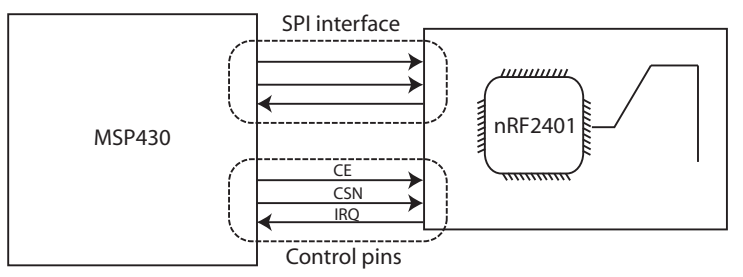

(a)

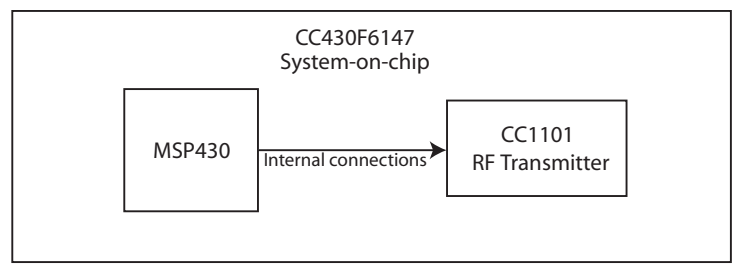

(b)

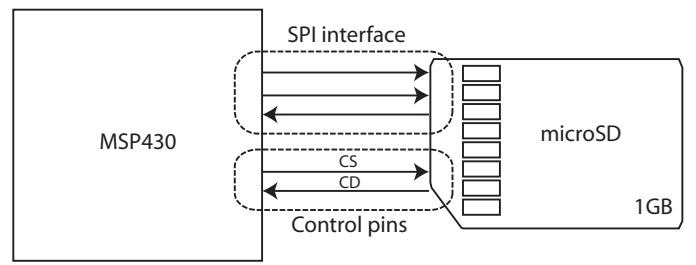

(c)

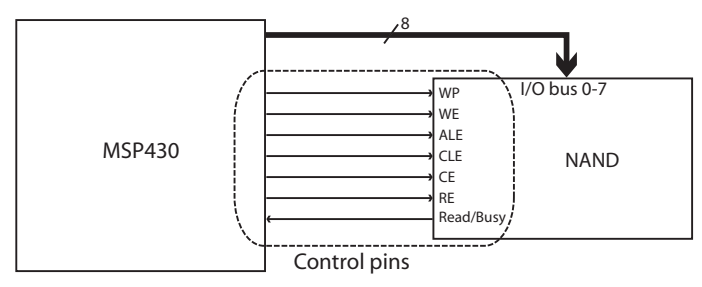

(d)

Fig. 1. Physical set up of the prototype sensor nodes with the four different back-ends. (a) Nordic transmitter; (b) CC430 transmitter; (c) microSD card; (d) NAND flash memory chip.

\section{4) A Hynix NAND flash memory.}

Wireless node topologies ( 1 and 2 ) provide flexibility, ease of use, and allow real-time access to the collected signals. Local memory topologies ( 3 and 4 ) do not provide real-time access to the data being collected, but are still of significant interest as there is no risk of missing packets, and due to their low power operation. Generally, with no compression present, local memory based systems have a lower average current consumption than wireless systems and this is demonstrated here in Section IV.

The wireless nodes are set up as:

1) Nordic nRF2401+: Our prototype wireless node uses a Nordic Semiconductor nRF2401+ transceiver [19] set with a transmit power of $0 \mathrm{dBm}, 2 \mathrm{Mbps}$ over-the-air data rate, and $2.4 \mathrm{GHz}$ operation frequency. Each time a frame of samples is passed to the transmitter by the MSP430 the data is transmitted as quickly as possible over the $2 \mathrm{Mbps}$ link with the transmitter turned off the rest of the time to save power. The Nordic Shockburst protocol is used with a 32 byte payload size and five preamble, address and CRC (Cyclic Redundancy Check) bytes. It operates from a $2 \mathrm{~V}$ supply. 
2) TI CC430 wireless transmitter: The CC430 node is based upon the CC430F6147 [20] which incorporates both an MSP430 core and a CC1101 sub-GHz wireless transceiver. It is set to operate at $868 \mathrm{MHz}$, with a transmit power of $0 \mathrm{dBm}$, 64 byte payload size and three preamble and CRC bytes. This node is powered using a $3 \mathrm{~V}$ supply.

The local memory sensor nodes are set up as below. In these there is a choice of both the type of memory and the file system used. All microSD cards require a file system and we use FAT16 for universal support across many commercial PCs with integrated card readers, making additional reader software unnecessary. In contrast our NAND memory node uses no standardised file system with data stored as simple binary files. The absence of a file system simplifies the data writing process and reduces the processing and memory burden on the microcontroller during the collection of data. This simplicity comes at the cost of more complexity when passing the data to an interpreting computer, but the sensor node can generally be powered by the interpreting computer during these operations.

3) microSD card: The microSD based sensor makes use of a class 4 microSD card and the MSP430 implements a customised version of the freely available Fatfs library [21] to access it. This allows cards up to 2 GB in size to be written to, enough space for at least a day of continuous operation. As more compression is employed in the system this duration will increase. A $3.3 \mathrm{~V}$ supply is required.

4) Hynix NAND flash memory: The NAND sensor node uses a Hynix NAND HY27UF081G2A flash chip [22]. This is a standard large block sized NAND device and so is representative of all large memory NAND units, although the precise model used at present only has a $128 \mathrm{MB}$ capacity. It operates from a $2.7 \mathrm{~V}$ supply.

\section{B. Compression design}

The MSP430 is responsible for the set up and access of the back-end, and for performing low power digital compressive sensing to minimise the amount of data that needs to be transferred. For each input signal $\mathbf{x}$-in our case an EEG channel recorded from the human scalp-a compressively sensed representation is generated in the digital domain by carrying out the matrix multiplication

$$
\mathbf{y}=\boldsymbol{\Phi} \mathbf{x} \text {. }
$$

Here $\mathbf{x}$ is a non-overlapping frame of $N$ EEG samples, and $\mathbf{\Phi}$ is an $M \times N$ sensing matrix. $\mathbf{y}$ has dimensions $M \times 1$ and so if $M<N$ data compression is achieved. Provided that $\boldsymbol{\Phi}$ is correctly chosen, reconstruction of $\mathbf{x}$ from $\mathbf{y}$ is possible even though $\mathbf{y}$ has fewer samples than a signal sampled at the Nyquist rate. It is this vector $\mathbf{y}$ that is actually stored in the local memory or wirelessly transmitted from the sensor.

In either case, once the samples of $\mathbf{y}$ have been passed to a receiving computer the original vector $\mathbf{x}$ needs to be reconstructed so that the original EEG signal can be inspected and used. This reconstruction is carried by solving the optimization problem

$$
\begin{gathered}
\min _{\mathbf{S} \in \Re^{N}}\|\mathbf{s}\|_{l_{1}} \text { subject to } \mathbf{y}=\mathbf{\Phi} \mathbf{\Psi} \mathbf{s} \\
\mathbf{r}=\mathbf{\Psi} \mathbf{s}
\end{gathered}
$$

where $\Psi$ is a basis function in which the input $\mathbf{x}$ can be represented sparsely as $\mathbf{s}(\mathbf{x}=\mathbf{\Psi} \mathbf{s})$. That is, most entries in $\mathbf{s}$ should be zero, or near zero. $\mathbf{r}$ is then the reconstructed estimate of the original input $\mathbf{x}$.

The key criteria for successful compressive sensing are that $\Phi$ and $\boldsymbol{\Psi}$ are incoherent and that $\mathbf{s}$ is a good sparse representation of the signal [7], [8]. The first condition is satisfied by drawing entries in $\Phi$ from a random distribution. $\Psi$ can then be any suitable choice for obtaining a sparse $\mathbf{s}$, and it can be changed during the reconstruction stage; knowledge of $\boldsymbol{\Psi}$ is not required during the initial sampling process. Depending on the precise choices for $\boldsymbol{\Phi}, \boldsymbol{\Psi}$, and the reconstruction minimisation method, many different compressive sensing implementations are possible with differing reconstruction performances and characteristics.

To minimise the sensor node power consumption we use a Bernoulli distribution, $p=0.6$ with values of only 0 and 1 for $\Phi$. This $\Phi$ reduces the matrix multiplication (1) to an accumulation, greatly reducing the processing load. The fixed point output is accumulated into the MSP430 16 bit registers avoiding any potential overflow. On-the-fly generation of random numbers is an intensive process and to reduce the processing load we use non-adaptive compressive sensing [13] where a fixed sampling matrix is used for each frame, with the same matrix used for all EEG channels. This matrix is generated a priori in MATLAB via the randn function and stored in the local flash memory of the MSP430.

For the signal reconstruction, which is carried out on a standard desktop computer, we use a Basis Pursuit optimization procedure [23], [24] with a cubic B-spline dictionary [25], [26] for $\Psi$. These settings correspond to the best reconstruction case for EEG signals as reported in [12].

\section{Other system aspects}

All nodes are set up assuming the collection of 16 channels of $200 \mathrm{~Hz}$ sampled EEG data. To provide a fair comparison platform, front-end signal conditioning and analogue-to-digital conversion affects all cases uniformly and so is not included in the analysis here which measures only the current consumption of the MSP430 and back-end unit. Suitable front-end systems for EEG applications include [4], [27] and a complete review of low power ADCs is in [28]. Instead of the front-end blocks, 16 channels of pre-recorded EEG data are stored in signed 16 bit fixed-point format (Q3.12) in the internal MSP430 flash memory. This is loaded a sample at a time into the MSP430 and once $N$ samples from all 16 channels have been loaded the compressive sensing operation is performed. This produces $M$ compressively sensed samples per channel which are passed out of the MSP430 to the transmitter/memory unit. The MSP430 can operate with a supply voltage anywhere between $1.8 \mathrm{~V}$ and $3.6 \mathrm{~V}$. To minimise the power consumption, and to require only one regulator in each system, this voltage is set to match requirements of the back-end being used, as given in Section II-A.

\section{ANALYSIS METHODS}

In this article we investigate a number of different factors affecting the performance of compressive sensing in wearable 


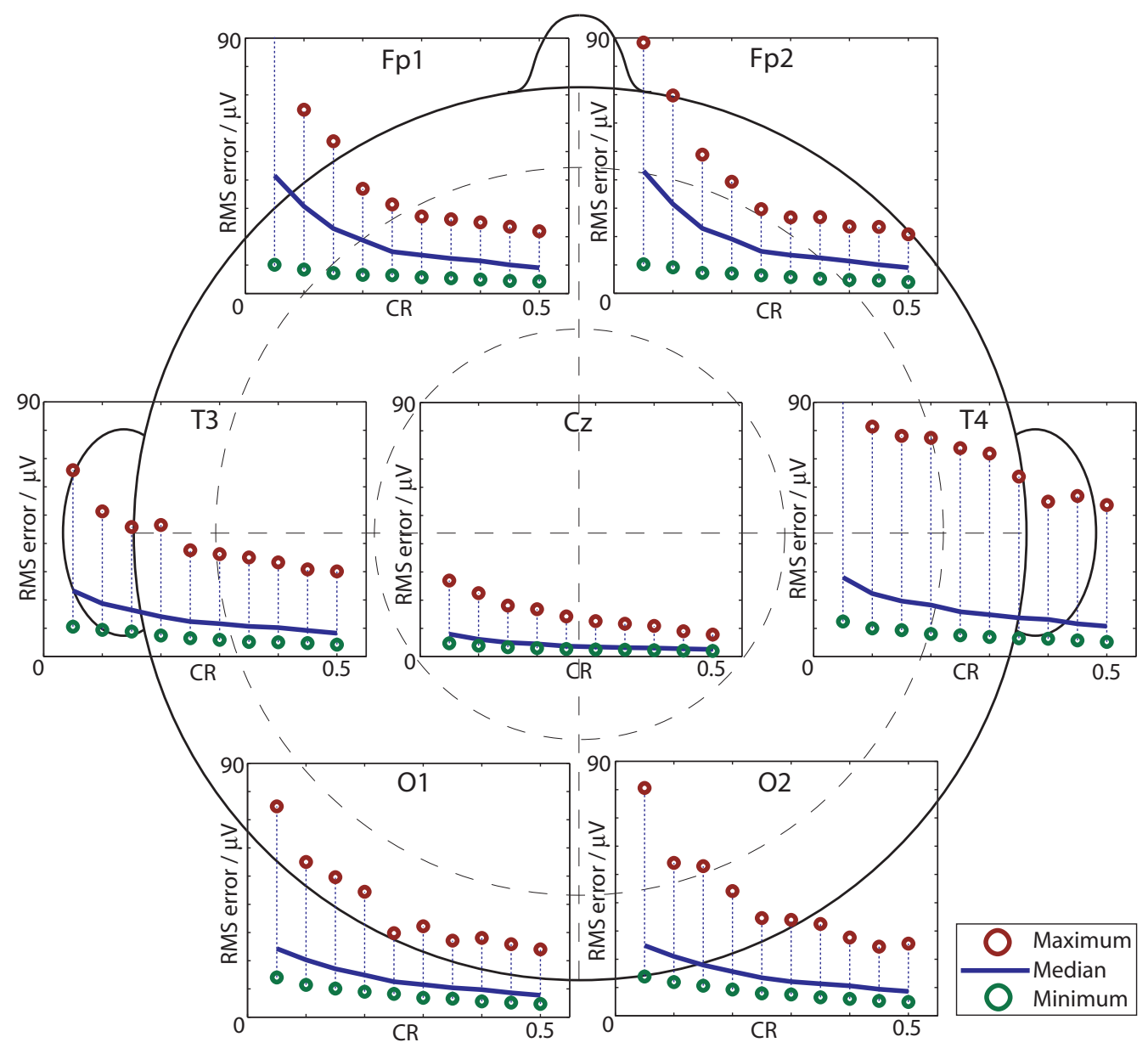

Fig. 2. Reconstruction performance of the fixed point compressive sensing scheme as the compression ratio $(C R)$ is varied. Values for the median, minimum and maximum RMS error values found from analysing 30 minutes of EEG data are shown. Results are arranged for each EEG channel as they are located on the head, and generated using frame size $N=200$.

sensor nodes. Firstly, we investigate the current consumption to quantify the power benefits of the on-sensor-node compression. The sensor node current is measured using a $500 \mathrm{MHz}$ oscilloscope and a $10 \Omega$ sense resistor placed in series between the power supply and the system. This allows the current to be measured as the system moves through the stages: MSP430 active and doing compressive sensing; MSP430 and back-end active and writing/sending out data; and the MSP430 and backend idle. The total average current is then easily calculated.

Secondly, we investigate the reconstruction accuracy allowing us to demonstrate that the input physiological signal can be accurately recorded. We used scalp EEG signals as the example application for this. While a number of studies have investigated the reconstruction performance of compressive sensing applied to EEG signals previously [12], [29]-[32] these do not take account of the compressive sensing hardware platform and its constraints. In particular, our own work [12] presented a comprehensive evaluation of the reconstruction performance of multiple compressive sensing implementations for scalp EEG signals. It is not the intention here to repeat this analysis. Instead, it is noted that [12] used a fully MATLAB based, floating point, implementation of compressive sensing.

Thirdly we therefore verify here that a fixed point imple- mentation suitable for the MSP430 is not substantially worse than the previous floating point one. Our input EEG signals are stored offline as 16 bit EDF files [33]. These are converted for a 16 bit fixed-point compressive sensing implementation and we present results for the reconstruction error using the fixed point implementation, and compare these to the fully MATLAB based implementation used in [12]. The reconstruction and error calculation procedures are the same for both, only the compressively sensed samples change. Using the fixed point toolbox MATLAB can accurately simulate both the floating and fixed point compressive sensing implementations and the performance is found offline, not by connecting sensor nodes to subjects. Although the MSP430 based compressive sensing assumes a full 16 channel EEG system, for compactness and visualisation here we present reconstruction results for only seven channels. These are Fp1, Fp2, T3, Cz, T4, O1, O2 all recorded at $200 \mathrm{~Hz}$ with an $\mathrm{FCz}$ reference. Thirty minutes of data from one subject are used.

The reconstruction performance is characterised through the RMS (Root-Mean-Square) error calculated using $10 \mathrm{~s}$ of reconstructed data, allowing maximum, minimum and median values over time to be found. The reconstruction performance was also characterised through the PRD (Percentage RMS 
Difference) error and similar trends were observed. All of these performance measures are calculated for a range of compression ratios $(C R)$ and frame sizes $(N)$ where

$$
C R=\frac{M}{N} \text {. }
$$

Lower $C R$ values represent more data compression. This allows us to investigate the optimal compressive sensing set up for each sensor node topology as a function of compression ratio $C R$ and frame size $N$.

\section{SYSTEM PERFORMANCES}

\section{A. Reconstruction accuracy}

Fig. 2 shows the reconstruction performance of the fixed point compressive sensing across the different regions of the head. It can be seen that reconstruction is possible, with the general result similar across all channels. As observed in [12], better performance is achieved along the midline ( $\mathrm{Cz}$ here) and the performance is approximately symmetric across the midline except in T3/T4 where higher maximum errors are seen in T4. This occurs due to a period of asymmetric EMG artifact in the EEG (with more artefact present on T4 than T3) most likely due to chewing/jaw action. Our test EEG data is taken from out-patient, ambulatory EEG recordings in uncontrolled environments to reflect real-world sensor node operation. As such our results demonstrate the absolute performance bounds obtained during prolonged free-running EEG recording. In some use cases, if the entire free-running EEG is not wanted and artefact sections can be removed, our results are thus a pessimistic bound.

In all cases in Fig. 2 as the compression ratio is reduced the median, max and min of errors all get worse, and the impact of frame size $N$ on this is shown in Fig. 3. Here, the larger $N=200$ frame size performance is slightly better, but it is not a large effect. The reconstruction performance is principally only a function of the compression ratio.

\section{B. Comparison to floating point}

The differences in RMS error between the MATLAB simulated fixed point and floating point implementations are shown in Fig. 4 for two channels. Channel $\mathrm{Cz}$, which achieved the best overall performance in Fig. 2, now achieves the worst performance of all of the analysed channels, although the differences are never large. In addition, in $\mathrm{Cz}$ there is a strong correlation between the compression ratio and how well the two implementations match: smaller compression ratios have less error between them. This effect is not seen in any of the other channels where the error is essentially independent of the compression ratio used. The origin of this is currently unknown but highlighted here as a systematic effect which may become significant to future researchers. It is noted that $\mathrm{Cz}$ is the closest electrode to the reference at $\mathrm{FCz}$, but it is not clear why this would systematically impact the fixed-point vs floating-point operation of the compressive sensing.

Overall from Fig. 4 it is clear that the use of a fixed point implementation has no meaningful impact on the compressive sensing performance. As with the reconstruction accuracy

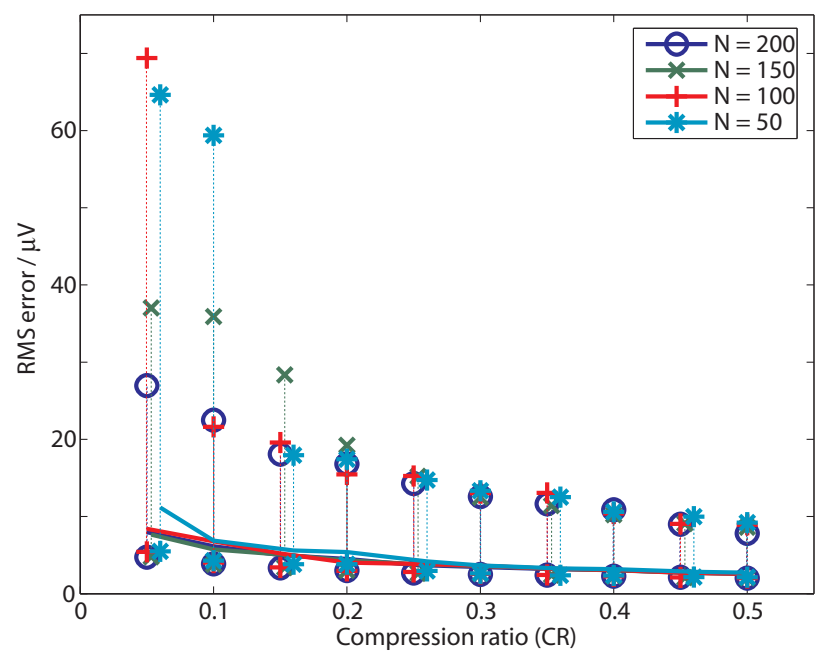

(a)

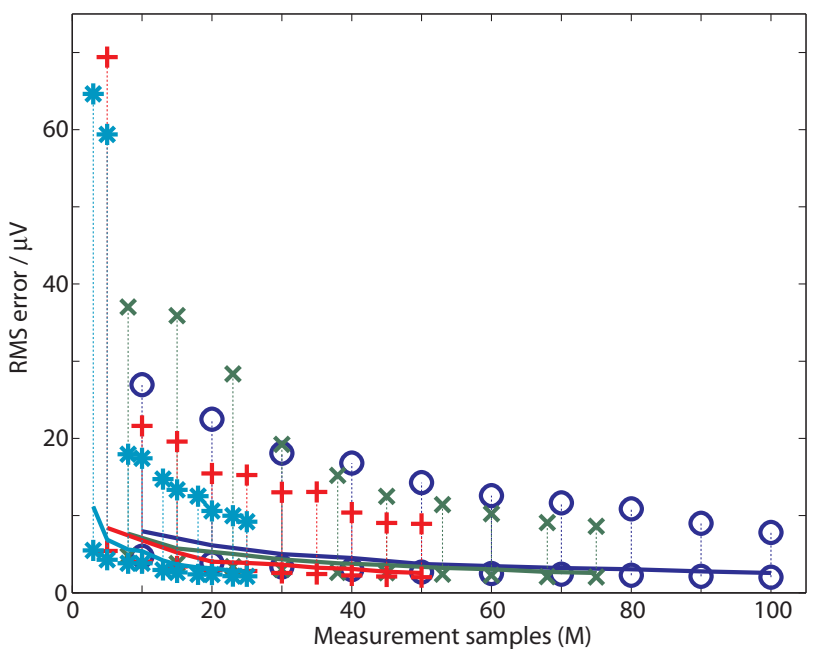

(b)

Fig. 3. Reconstruction performance of the fixed point compressive sensing scheme in channel $\mathrm{Cz}$ using four different frame sizes $(N)$. Each vertical line plots the median, minimum and maximum RMS error found from analysing 30 minutes of EEG data. (a) against the compression ratio $(C R)$; (b) against the number of compressed samples $(M)$.

results the frame size used has little consistent impact on reconstruction.

\section{Peak current consumption}

For classical systems with high average current draws, or which use physically large batteries, the average current consumption of the system is the only power consumption factor of interest. However many physically small batteries, in addition to having low total amounts of energy storage have low maximum peak draws [34]. For low power systems such as ours the peak current can thus be the limiting factor determining the battery size, not the energy density. Hence the measured peak currents from the entire system, and their durations, are given in Table I for the four sensor nodes. The maximum current ever drawn by MSP430 alone is also listed.

The maximum current drawn by the MSP430 alone (3.8 $\mathrm{mA})$ is much smaller than that drawn when both the 


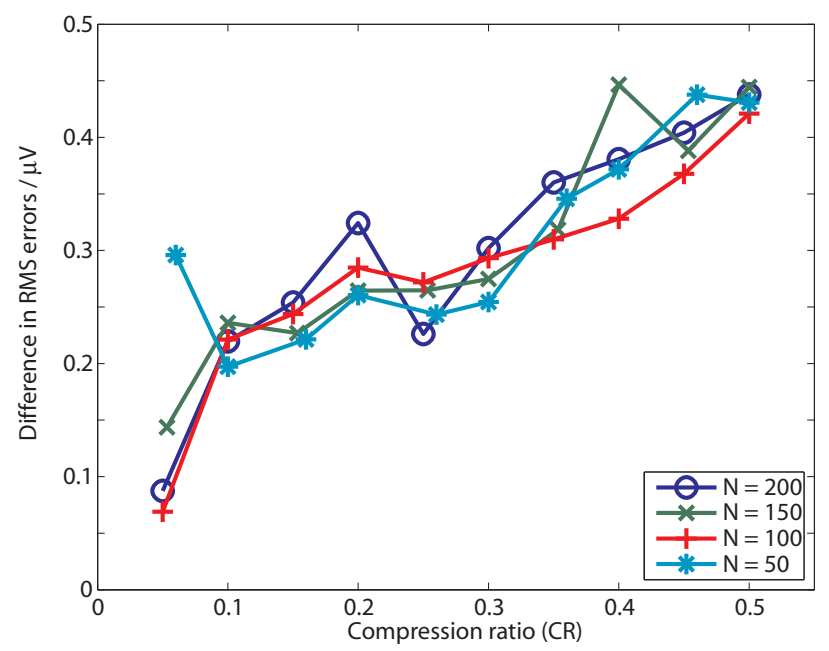

(a)

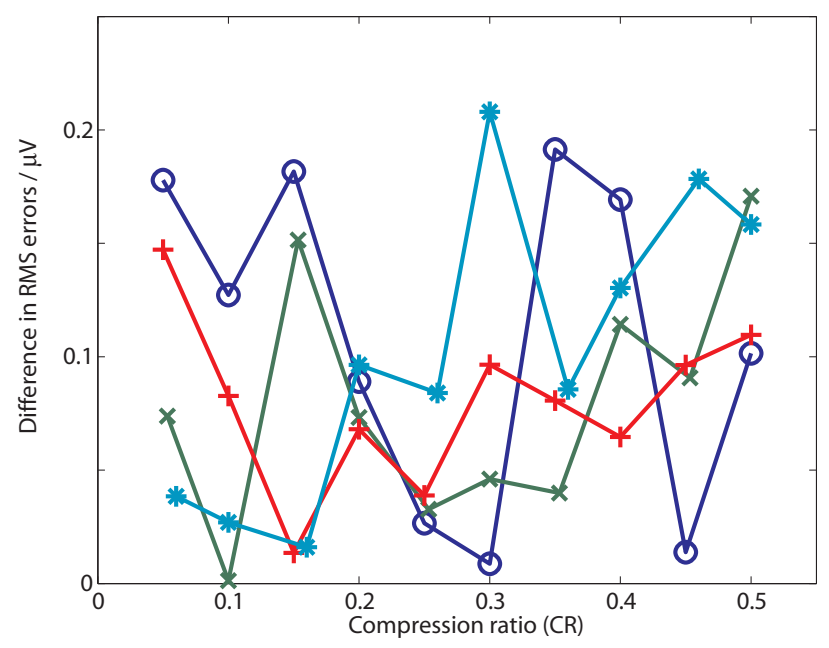

(b)

Fig. 4. Difference in the RMS reconstruction errors between the fixed point MSP430 suitable and fully floating point compressive sensing implementations for different frame sizes $(N)$. (a) in channel $\mathrm{Cz}$; (b) in channel $\mathrm{O} 2$.

TABLE I

PEAK CURRENTS AND THEIR DURATION FOR THE MSP430 COMPRESSIVE SENSING AND FOR THE COMPLETE SYSTEM WITH FOUR DIFFERENT BACK-ENDS.

\begin{tabular}{lcc}
\hline Operation & $\begin{array}{c}\text { Peak current } \\
\text { draw / mA }\end{array}$ & Duration / $\mu \mathrm{s}$ \\
\hline $\begin{array}{l}\text { MSP430 compressive sensing } \\
\text { (1 MHz clock) }\end{array}$ & 0.32 & - \\
MSP430 compressive sensing & 3.8 & - \\
(16 MHz clock) & 14 & 205 \\
Nordic wireless transmitter & 18 & 220 \\
CC430 wireless transmitter & 57 & 250 \\
microSD card & 19 & 230 \\
NAND flash memory chip & & \\
\hline
\end{tabular}

MSP430 and back-end are considered (which varies from 14$57 \mathrm{~mA}$ ). We can therefore clock the MSP430 at $16 \mathrm{MHz}$ where it is more efficient per clock cycle to give a lower average power consumption. Ordinarily such operation might have be avoided if the increase in peak current was not acceptable. Similarly, both wireless back-ends have lower peak current requirements than the local memory units, highlighting a further potential advantage of moving to wireless sensor node topologies in low power, low size sensor nodes.

Typical timing diagrams for the four sensor nodes are shown in Fig. 5 illustrating very different current profiles. For the wireless transmitters distinct bursts are present corresponding to the transmission of individual packets. These are interspersed with the compressive sensing of the input signals and a small amount of set up time for the transmitter. In contrast for both the NAND memory and microSD card there is a broad increase in current as all of the data is written out with local peaks present during the card access times. The microSD card takes considerably longer to write 50 samples, approximately $2.5 \mathrm{~ms}$. The Nordic and CC430 transmitters require approximately $1.5 \mathrm{~ms}$ and $1.2 \mathrm{~ms}$ respectively while the NAND flash memory only $300 \mu \mathrm{s}$.

\section{Average continuous current draw}

Assuming that the battery used can provide the required peak current, the average continuous current consumption is the main power design factor. This current requirement is shown for the four nodes in Fig. 6 as a function of the compression ratio used. Horizontal lines show the power consumption of the system when no compression is present and all of the input data is sent to the back-end. For compressive sensing to be power beneficial the current consumption with compressive sensing turned on must be below these lines.

When no compression is present is it clear that it is beneficial to process the data in as large a frame size as possible, with larger frame sizes always requiring the least current. In line with the traditional motivation for the use of local memory sensor nodes, in the no compression case the average current of the Nordic system is approximately $1 \mathrm{~mA}$, whereas for the NAND system it is only $0.2 \mathrm{~mA}$, nearly an order of magnitude lower.

However compressive sensing is much more beneficial for the wireless transmitter cases (Fig. 6(a) and Fig. 6(b)) than for the local memory cases. In particular for the microSD card, although there are cases where the use of compressive sensing is beneficial, the improvement in current consumption is in general small. We find that it is the setting up of the file system that dominates the system power consumption, not the process of actually writing data. The frame size $N$ used therefore dominates the system design: if $N$ is large the file system is set up, addressed and accessed fewer times for writing out the same amount of data.

In both wireless systems, compressive sensing decreases the system current requirements significantly, and at the lowest compression ratios it can be brought to the same power ranges as the local memory topologies. Moreover, with the wireless transmitters compressive sensing is power beneficial for essentially all of the frame sizes and compression ratios used. In 


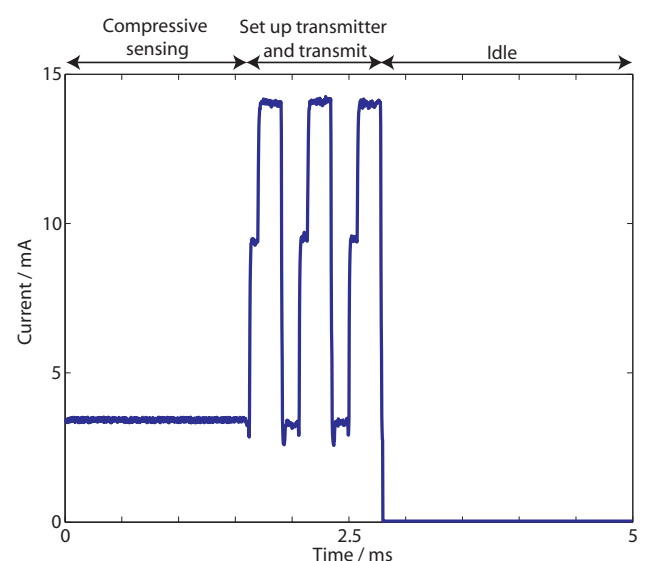

(a)

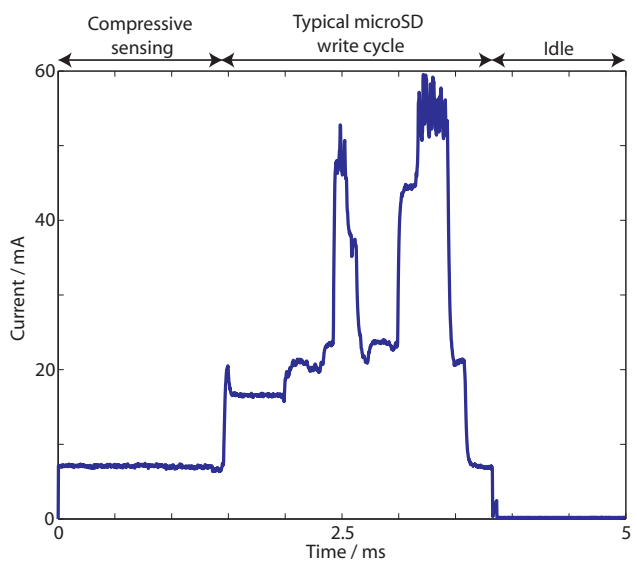

(c)

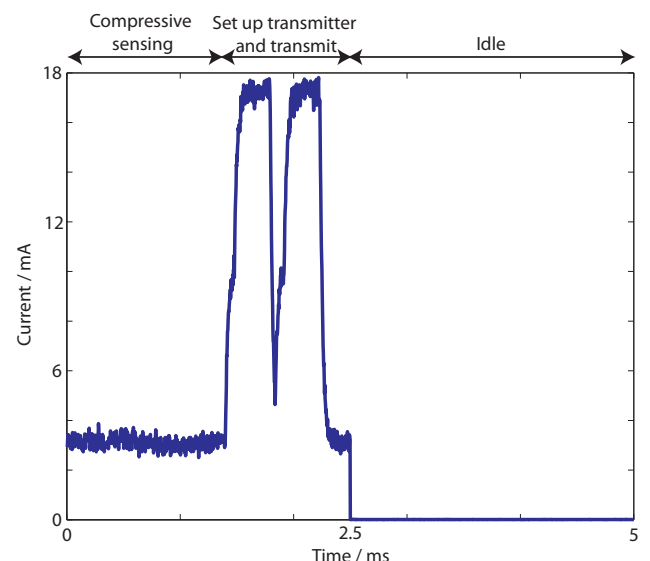

(b)

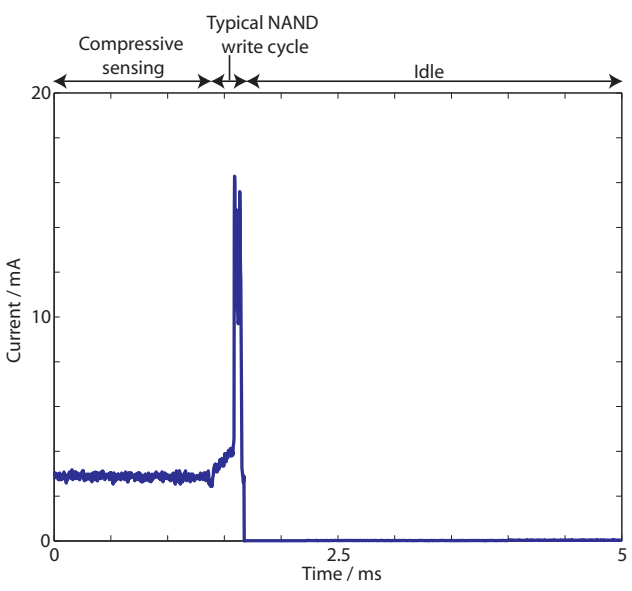

(d)

Fig. 5. Current measured during a compress, transmit/store, idle cycle of the sensor node for $M=50, N=100$. Only a portion of the compressive sensing period is shown. Actual duration is $31 \mathrm{~ms}$ (for 16 channels) and the remainder of the cycle is spent in the idle state. (a) Nordic transmitter; (b) CC430 transmitter; (c) microSD card; (d) NAND flash memory chip.

all cases the lowest absolute power consumption is achieved by using larger frame sizes. However the system needs to operate at the lowest compression ratios to achieve this and the increase in the reconstruction error (Fig. 2) must be tolerated. For more practical compression ratios in the $0.2-0.5$ range, using smaller frame sizes is a more appropriate design strategy. For the wireless transmitter at these compression ratios it is the smaller frames that give the lowest current consumption and correspondingly the largest percentage improvement in current consumption.

\section{E. Relative current consumptions}

The motivation for on sensor node data compression is due to the presence of a high power transmitter/storage stage. This dominates the power consumption and thus expending some power on data compression to give less data to transmit can remove this dominance and lead to overall power savings [2][4]. To evaluate the impact of compressive sensing on this Fig. 7 breaks down the measured average current values from Fig. 6 into the three main stages of operation: system idle, system performing compressive sensing, and transmission/storage of the data; and shows the percentage of the current used during each stage. For clarity only results for two frame sizes, $N=50$ and $N=200$, are shown.

To minimise power consumption the sensor nodes have been designed to spend as much time as possible in the idle mode and this results in a considerable amount (up to $20 \%$ ) of the total system current being used in this mode. Indeed the sensor node with the highest absolute consumption (the CC430 node) is the one which spends the minimum amount of power in idle. For the Nordic transmitter (Fig. 7(a)) at $N=200$ the transmission and the compressive sensing both use approximately equal percentages $(\sim 50 \%)$ of the total current at all compression ratios and so the transmitter dominance has been reduced compared to the compression costs. Nevertheless lower absolute consumptions are achieved at lower compression ratios due to the substantial increases in idle time possible as less data passes through the system.

In all of the cases in Fig. 7 there is a direct trade-off with the frame size $(N)$ used: large frames push towards using proportionally more current on compression, while smaller frame sizes push towards transmission/storage. Minimum power realisations of compressive sensing can thus use this to optimize the system power consumption without substantial impacts on the reconstruction performance (Fig. 2). This minimum power 


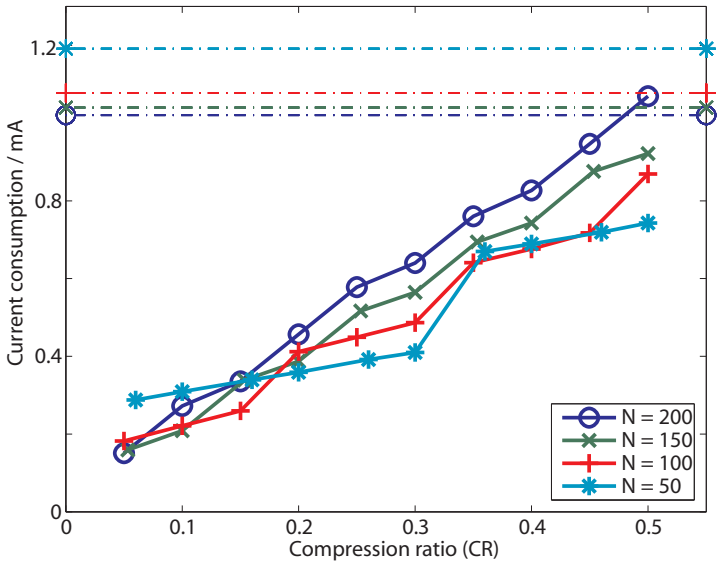

(a)

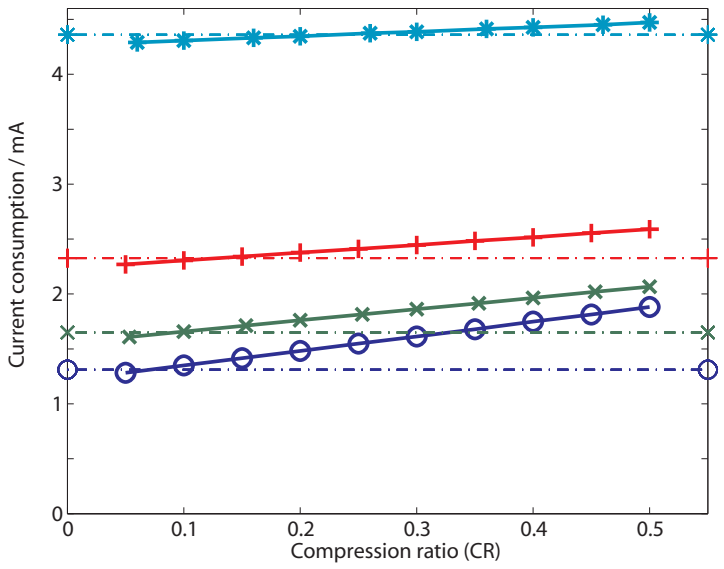

(c)

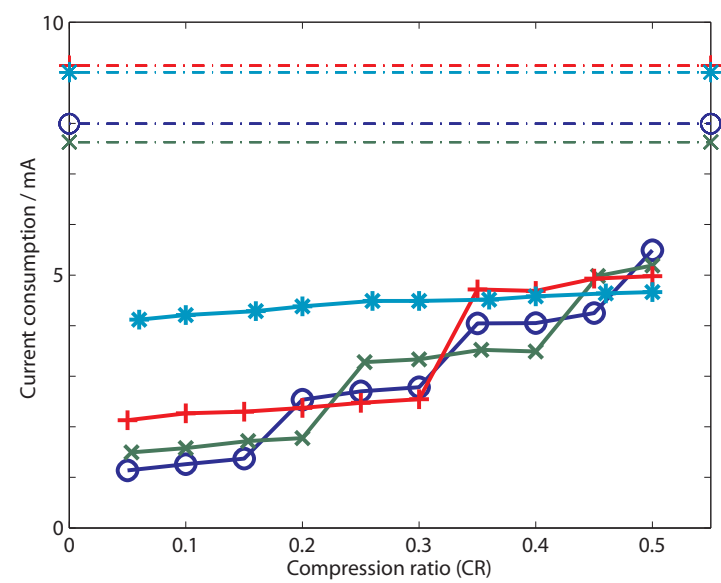

(b)

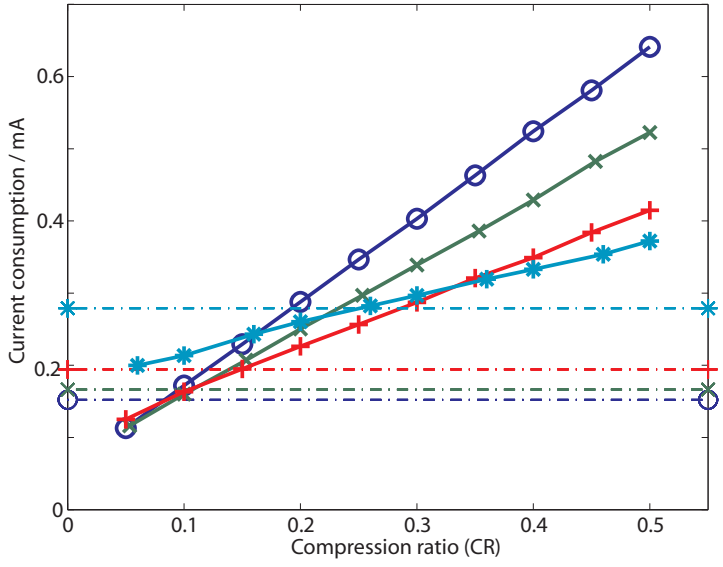

(d)

Fig. 6. Average continuous current draw for the three sensor nodes. Horizontal lines show the current consumption in the direct sampling cases when no compressive sensing is used and all data is passed to the back-end. (a) Nordic transmitter; (b) CC430 transmitter; (c) microSD card; (d) NAND flash memory chip.

realisation is best achieved at low compression ratios. However for the local memory sensor nodes, and the microSD card in particular, the percentage of current used for storage actually increases as more data reduction is provided.

\section{DISCUSSION AND CONCLUSIONS}

Low power data compression is an essential part of wearable sensor nodes for monitoring the human body. Compressive sensing is an emerging technique for use in these and it has shown utility in a number of physiological sensing applications [9]-[14]. Recent results have also suggested that compressive sensing applied within the sensor node could relax the noise specification for the front-end amplifier [6], provide a level of encryption [31], or potentially allow the number of bits in the analogue-to-digital converter to be reduced [35].

However compressive sensing is a lossy compression technique and although the requirement for minimum power implementations is well established, its performance is often only assessed in terms of reconstruction accuracy [14]. [36] presented a compressive sensing implementation using a MSP430 micro-controller and produced a complete working wireless ECG sensor. [6] presented a fully custom implementation of the digital multiplier required for the sampling process and provided the first fully on-chip implementation of sensor node compressive sensing. These however focused on the power minimisation of the compressive sensing stage only. The motivation for on sensor node data compression is due to the presence of a high power transmitter/storage stage and its power changes under different compressive sensing arrangements must also be accounted for. This paper has therefore investigated compressive sensing in terms of both reconstruction performance and power consumption taking into account different back-end technologies. Four different sensor nodes have been presented comprising of both wireless and local memory topologies.

Local memory sensor nodes are often used as, without on-node data compression, they have lower average current draws than wireless nodes. Our NAND memory based system required nearly an order of magnitude less current than the wireless transmitter systems. However wireless data collection is highly advantageous as it allows real-time access to the data. Our results showed that both wireless systems benefited substantially from the use of compressive sensing and in the Nordic transmitter case it successfully brought down the average current consumption to a comparable level to the NAND system. 


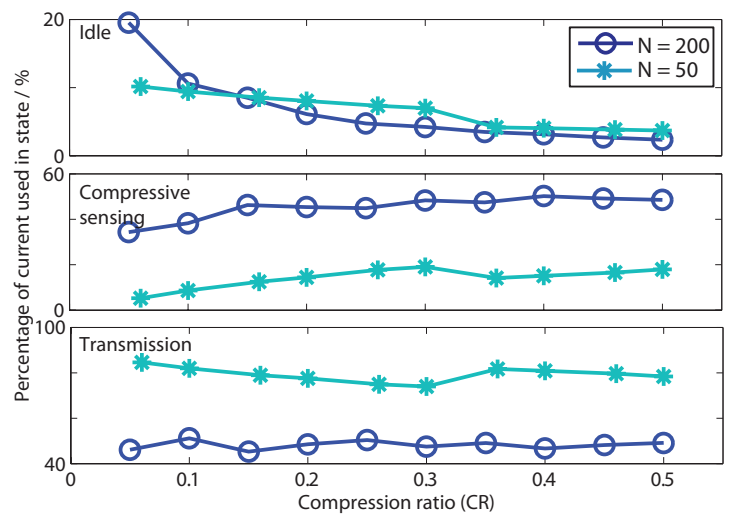

(a)

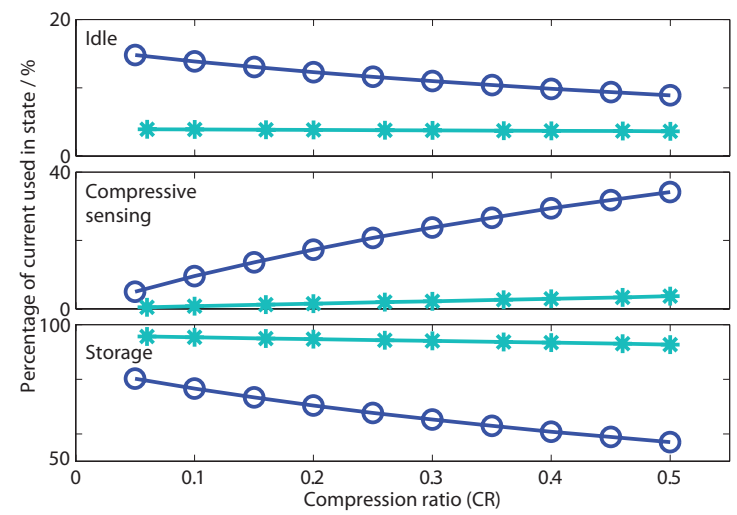

(c)

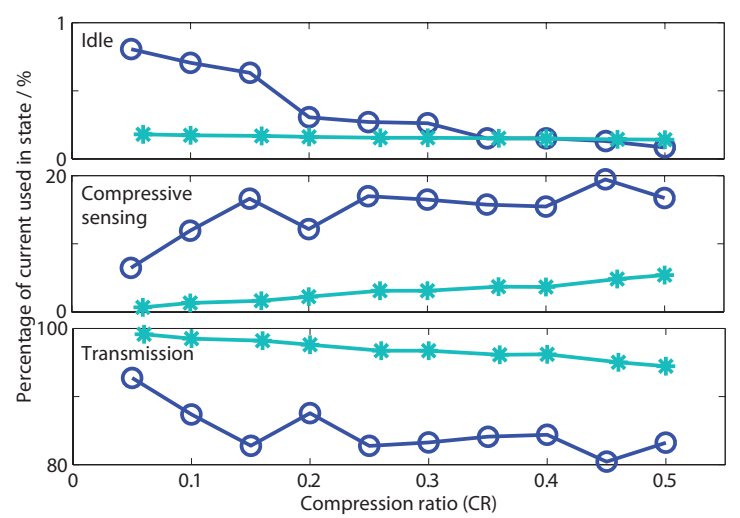

(b)

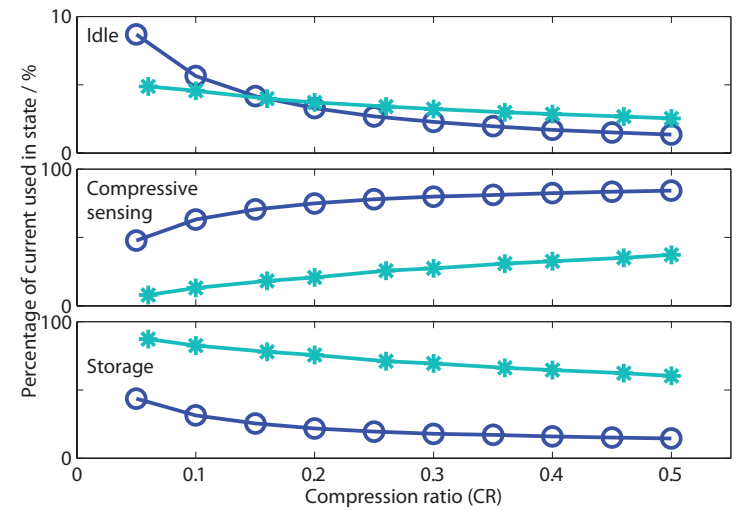

(d)

Fig. 7. Break-down of the current consumptions from Fig. 6 showing the percentage of the total consumption used in each of the main stages of operation. (a) Nordic transmitter; (b) CC430 transmitter; (c) microSD card; (d) NAND flash memory chip.

Therefore, assuming that the reconstruction error of the compressive sensing can be tolerated for the given application, there is now no intrinsic reason to prefer local storage based sensor nodes from a current consumption point of view. Indeed, once the average consumption of the wireless system is comparable to the NAND, the overall system can benefit from the lower supply voltage required by the wireless transmitter and the lower peak current requirement. Both of these allow the use of physically smaller batteries to power the overall system. This is made possible because the raw EEG data rate is only $25.6 \mathrm{kpbs}$ and is low compared to the $2 \mathrm{Mbps}$ data rate of the wireless transmitter and so it can be significantly duty cycled. Although we have used 16 channel EEG data as our example application, this comparatively low data rate will also apply to many other physiological parameters such as the EOG, EMG and ECG.

The reconstruction error was found to be independent of whether a fixed or floating point compressive sensing implementation was used, and also independent of the frame size $N$. As a result, considering the signal processing perspective only these are free choices. When combined with the current consumptions presented here however the power consumption is strongly dependent on the frame size and the back-end technology used. Indeed the frame size controls the power balance present in the system: larger frames shifted power towards compression while smaller sizes shifted power towards transmission. Far from a free choice, this is therefore a critical parameter for optimizing the compressive sensing power consumption, and compressive sensing schemes for sensor nodes must be evaluated in terms of both reconstruction and power performance. We have illustrated this power tradeoff for four different types of back-end.

Inevitably there are other practical factors in addition to the current consumption to consider when selecting between the use of wireless sensor nodes and local memory sensor nodes. For example, the wireless sensor nodes use a minimum latency transmission scheme where data is sent as soon as possible to keep the system real-time. It is possible to potentially reduce power consumption further by choosing a transmission scheme where data is accumulated for some time and then sent out in larger packets. This, and other techniques for transmitter power optimization are discussed in [37]. It is also possible to use a memory card in series with a wireless transmitter to buffer the data before transmission if latency can be tolerated in the node. Further, the regulations governing transmit power and interference are much more stringent for wireless nodes, and the impacts of bit errors and missing packets need to be accounted for. For local memory systems, the microSD node required a FAT16 file system and the set up of this dominated the power consumption, not the process of actually writing out data. The NAND memory node did not use this to save power, but as a result the memory cannot be directly read by a host PC without requiring additional software, impacting the ease of use. Finally our results do no control for the 
semiconductor process size used in the microSD card and NAND memories. While the general form of our results will be unchanged, this parameter will affect the precise currents drawn by these memory units, with smaller process nodes requiring less current. Unfortunately process node information is not released by the memory manufacturers.

Nevertheless our results give a practical overview of the performance of state-of-the-art sensor node systems and we believe the investigations will be highly informative for others selecting between the different sensor node topologies. For optimizing the compression implementation, for practical compression ratios in the $0.2-0.5$ range smaller frame sizes offer lower current consumption, and a greater percentage improvements in the current consumption, although the lowest absolute current consumptions were achieved with larger frame sizes. The reconstruction error was independent of this and so the compressive sensing design must be governed by the back-end considerations; it is not possible to consider the compression design and sensor topology design in isolation.

\section{REFERENCES}

[1] P. Bonato, "Wearable sensors and systems," IEEE Eng. Med. Biol. Mag., vol. 29, no. 3, pp. 25-36, 2010.

[2] A. J. Casson, D. C. Yates, S. J. Smith, J. S. Duncan, and E. RodriguezVillegas, "Wearable electroencephalography," IEEE Eng. Med. Biol. Mag., vol. 29, no. 3, pp. 44-56, 2010.

[3] B. Gyselinckx, C. Van Hoof, J. Ryckaert, R. F. Yazicioglu, P. Fiorini, and V. Leonov, "Human++: autonomous wireless sensors for body area networks," in IEEE CICC, San Jose, September 2005.

[4] N. Verma, A. Shoeb, J. Bohorquez, J. Dawson, J. Guttag, and A. P. Chandrakasan, "A micro-power EEG acquisition SoC with integrated feature extraction processor for a chronic seizure detection system," IEEE J. Solid-State Circuits, vol. 45, no. 4, pp. 804-816, 2010.

[5] A. M. Abdulghani, A. J. Casson, and E. Rodriguez-Villegas, "Quantifying the feasibility of compressive sensing in portable electroencephalography systems," in HCI international, San Diego, July 2009.

[6] F. Chen, A. P. Chandrakasan, and V. M. Stojanovic, "Design and analysis of a hardware-efficient compressed sensing architecture for data compression in wireless sensors," IEEE J. Solid-State Circuits, vol. 47, no. 3, pp. 744-756, 2012.

[7] D. L. Donoho, "Compressed sensing," IEEE Trans. Inform. Theory, vol. 52, no. 4, pp. 1289-1306, 2006.

[8] E. J. Candes and M. B. Wakin, "An introduction to compressive sampling," IEEE Signal Processing Mag., vol. 25, no. 2, pp. 21-30, 2008.

[9] U. Gamper, P. Boesiger, and S. Kozerke, "Compressed sensing in dynamic MRI," Magn. Reson. Med., vol. 59, no. 2, pp. 365-373, 2008

[10] T. V. Sreenivas and W. B. Kleijn, "Compressive sensing for sparsely excited speech signals," in IEEE ICASSP, Taipei, April 2009.

[11] Y. Zhang, S. Mei, Q. Chen, and Z. Chen, "A novel image/video coding method based on compressive sensing theory," in IEEE ICASSP, Las Vagas, April 2008.

[12] A. M. Abdulghani, A. J. Casson, and E. Rodriguez-Villegas, "Compressive sensing scalp EEG signals: implementations and practical performance," Med. Biol. Eng. Comput., vol. 50, no. 11, pp. 1137-1145, 2012.

[13] A. M. R. Dixon, E. G. Allstot, D. Gangopadhyay, and D. J. Allstot, "Compressed sensing system considerations for ECG and EMG wireless biosensors," IEEE Trans. Biomed. Circuits Syst., vol. 6, no. 2, pp. 156$166,2012$.

[14] Z. Zhang, T.-P. Jung, S. Makeig, and B. D. Rao, "Compressed sensing of EEG for wireless telemonitoring with low energy consumption and inexpensive hardware," IEEE Trans. Biomed. Eng., vol. 60, no. 1, pp. 221-224, 2013.

[15] Texas Instruments. (2012) MSP430 ultra-low power 16-bit microcontrollers. [Online]. Available: http://www.msp430.com/.

[16] H. Dubois-Ferrière, L. Fabre, R. Meier, and P. Metrailler, "TinyNode: a comprehensive platform for wireless sensor network applications," in Proc. IPSN, Nashville, April 2006.
[17] M. Johnson, M. Healy, P. van de Ven, M. Hayes, J. Nelson, T. Newe, and E. Lewis, "A comparative review of wireless sensor network mote technologies," in IEEE Sensors, Christchurch, October 2009.

[18] A. Burns, B. R. Greene, M. J. McGrath, T. J. O'Shea, B. Kuris, S. M. Ayer, F. Stroiescu, and V. Cionca, "Shimmer" $\mathrm{TM}$ - a wireless sensor platform for noninvasive biomedical research," IEEE Sensors J., vol. 10, no. 9, pp. 1527-1534, 2010.

[19] Nordic Semiconductor. (2012) Home page. [Online]. Available: http://www.nordicsemi.com/.

[20] Texas Instruments. (2013) CC430 RF SOC series. [Online]. Available: http://www.ti.com/lsds/ti/microcontroller/16-bit_msp430/rf_ soc/overview.page.

[21] FatFs Generic FAT File System Module. (2012) Home page. [Online]. Available: http://elm-chan.org/fsw/ff/00index_e.html.

[22] Hynix. (2012) Home page. [Online]. Available: http://www.hynix.com/.

[23] S. S. Chen, D. L. Donoho, and M. A. Saunders, "Atomic decomposition by basis pursuit," SIAM Rev., vol. 43, no. 1, pp. 129-159, 2001.

[24] SparseLab. (2012) Home page. [Online]. Available: http://sparselab. stanford.edu/.

[25] M. Andrle and L. Rebollo-Neira, "Cardinal B-spline dictionaries on a compact interval," Appl. Comput. Harmon. Anal., vol. 18, no. 3, pp. 336-346, 2005.

[26] Highly nonlinear approximations for sparse signal representation. (2012) Home page. [Online]. Available: http://www.nonlinear-approx.info/.

[27] J. Xu, R. Yazicioglu, P. Harpe, K. Makinwa, and C. V. Hoof, "A $200 \mu \mathrm{w}$ eight-channel acquisition ASIC for ambulatory EEG systems," in IEEE ISSCC, San Francisco, February 2011.

[28] B. Murmann. (2013) ADC Performance Survey 1997-2013. [Online]. Available: http://www.stanford.edu/ murmann/adcsurvey.html.

[29] S. Aviyente, "Compressed sensing framework for EEG compression," in IEEE/SP SSP, Madison, August 2007.

[30] S. Senay, L. F. Chaparro, M. Sun, and R. J. Sclabassi, "Compressive sensing and random filtering of EEG signals using slepian basis," in EUSIPC, Lausanne, August 2008.

[31] A. M. Abdulghani and E. Rodriguez-Villegas, "Compressive sensing: From "compressing while sampling" to "compressing and securing while sampling"," in IEEE EMBC, Buenos Aires, September 2010.

[32] Q. Hao and F. Hu, "A compressive eletroencephalography EEG sensor design," in IEEE Sensors, Kona, September 2010.

[33] B. Kemp and J. Olivan, "European data format 'plus' (EDF+), an EDF alike standard format for the exchange of physiological data," Clin. Neurophysiol., vol. 114, no. 9, pp. 1755-1761, 2003.

[34] G. Chen and E. Rodriguez-Villegas, "System-level design trade-offs for truly wearable wireless medical devices," in IEEE EMBC, Buenos Aires, September 2010.

[35] Igor Carron. (30th January 2010) CS: Q\&A with Esther RodriguezVillegas on a compressive sensing EEG. [Online]. Available: http://nuit-blanche.blogspot.com/.

[36] H. Mamaghanian, N. Khaled, D. Atienza, and P. Vandergheynst, "Compressed sensing for real-time energy-efficient ECG compression on wireless body sensor nodes," IEEE Trans. Biomed. Eng., vol. 58, no. 9, pp. 2456-2466, 2011

[37] A. Y. Wang, "Low power RF transceiver modelling and design for wireless microsensor networks," $\mathrm{PhD}$ thesis, Massachusetts Institute of Technology, 2005.

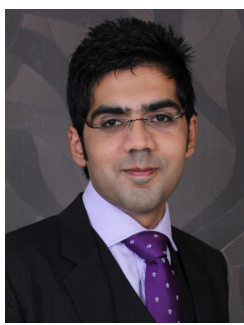

Syed Anas Imtiaz (S'07) received the B.Eng. degree from National University of Sciences and Technology, Pakistan in 2008 and M.Sc. degree with distinction in Integrated Circuit Design from Imperial College London, U.K. in 2009. He is currently working towards a Ph.D. degree in low power circuits and systems at Imperial College London. His research focuses on signal processing, algorithms and mixedsignal circuit design of low power and wearable EEG systems for use in sleep medicine and epilepsy monitoring. 
This is the author's version of an article that has been published in this journal. Changes were made to this version by the publisher prior to publication.

The final version of record is available at http://dx.doi.org/10.1109/TBME.2013.2293916

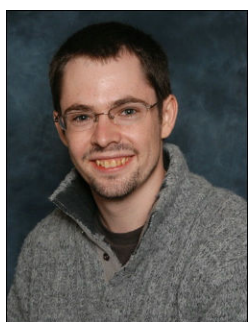

Alexander J. Casson (S'07, M'10) received the M.Eng. degree from the University of Oxford, U.K., in 2006 and the Ph.D. degree from Imperial College London, U.K. in 2010. He is currently a lecturer in the Sensing, Imaging and Signal Processing group in the school of Electrical and Electronic Engineering at the University of Manchester. His research focuses on creating innovative ultra-low power integrated circuits for brainwave monitoring, BCIs and transcranial stimulation. Previously Dr. Casson has worked on similar topics at Imperial College London and was the recipient of a prestigious Imperial College Junior Research Fellowship. His research has led to more than 40 publications and prizes including the Eryl Cadwaladr Davies prize for best doctoral thesis and an IET Ambition Award. Dr. Casson has also presented in high-profile minisymposiums at the IEEE Engineering in Medicine and Biology conference every year since 2010 .

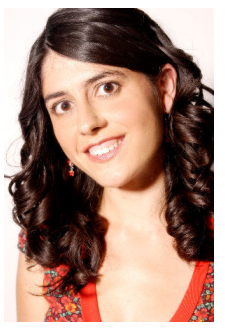

Esther Rodriguez-Villegas (SM'08) is currently a Reader in Low Power Electronics with the Department of Electrical and Electronic Engineering, Imperial College London, where she specializes in ultra-low-power electronic circuits and systems for truly wearable physiological monitoring. She is the author of more than 100 peer-reviewed papers and a book on FGMOS transistors which was published by the Institution of Engineering and Technology (IET) in 2006. Dr. Rodriguez-Villegas has been a member of technical committees in several international conferences such as the IEEE International Symposium on Circuits and Systems, the IEEE International Conference on Electronics, Circuits and Systems, and the IEEE Biomedical Circuits and Systems (BioCAS). In 2010 she became one of the European Research Council grantees and in 2009 she was awarded the Complutense Young Award for Science and Technology, and an IET innovation award. 